\title{
ASEGURAMIENTO DE LA CALIDAD Y DESARROLLO DE LA EDUCACIÓN SUPERIOR PRIVADA: COMPARACIONES ENTRE LAS EXPERIENCIAS DE ARGENTINA, CHILE Y URUGUAY ${ }^{1}$
}

\section{En el cruce de tendencias de la educación superior}

El establecimiento de mecanismos de aseguramiento de la calidad y el desarrollo de la educación superior privada son tendencias internacionales que se entrecruzan en la actualidad, resultando interesante observar sus interacciones.

Latinoamérica ha sido una de las regiones con mayor crecimiento de la educación superior privada. La literatura especializada explica este crecimiento por el aumento de la demanda y por las limitaciones del Estado para financiar las universidades públicas (Levy, 1986). En Chile, Brasil y Colombia el sector privado matricula más del 50\% de los estudiantes del sistema. En algunos países, donde el sector público no creció debido a exámenes de ingreso selectivos, el exceso de la demanda se transfirió al sector privado. En los países donde la masificación de las universidades públicas está dada por políticas de ingreso sin restricción, el sector privado se desarrolló con un carácter más de elite, contrastando con sus contrapartes del sector público (Schwartzman, 1994).

La educación terciaria privada es, en gran parte, orientada por el mercado (Altbach, 1999) y su desarrollo es, muchas veces, difícil de anticipar. En sistemas con larga tradición de monopolio público, la

1 Basado en el documento "Accreditation and Private Higher Education Development: A comparison between Argentina, Chile and Uruguay", presentado por los autores en la "2005 Annual Conference of the Association for the Study of Higher Education", Filadelfia, EE.UU., noviembre de 2005. 
diferenciación y el pluralismo provocados por las nuevas instituciones privadas producen sorpresa y, aun, perplejidad (Levy, 2002). Por esto, comprender el crecimiento privado es pertinente para la elaboración de políticas de educación superior. Experiencias de Argentina, Chile y Uruguay, así como de otros países, indican que la actitud del Estado es más bien reactiva al desarrollo privado, y no tanto el fruto de un desarrollo planificado.

Los mecanismos de aseguramiento de la calidad se han desarrollado internacionalmente como resultado del aumento de la demanda social por una rendición de cuentas (accountability) por parte de las instituciones de educación superior. La rápida expansión, tanto de instituciones como de programas académicos (incluyendo el dinámico sector privado), plantea la pregunta sobre la calidad de la oferta educativa. En América Latina, Centroamérica y Europa del Este, donde el crecimiento privado ha sido significativo en las últimas décadas, la acreditación se ha introducido como el instrumento clave de política para parte de los gobiernos, como forma de supervisar el crecimiento de esta modalidad educacional (Schwarz y Westerheijden, 2004). Este ha sido el caso de Argentina, Chile y Uruguay.

Muchos países, incluyendo los analizados en este estudio, distinguen entre acreditación y mecanismos de autorización de universidades privadas y sus programas. Los procesos de autorización inicial de universidades privadas reciben nombres diferentes en cada uno de los países: autorización en Argentina, licenciamiento en Chile y reconocimiento en Uruguay. La característica común es que una agencia estatal evalúa la propuesta de la institución universitaria privada y decide si se le permite ingresar al sistema o no. La autorización es un requisito y es obligatorio para otorgar los grados académicos; la acreditación es un mecanismo diseñado para verificar la calidad en el cumplimiento de estándares por programas ya autorizados o en instituciones que algunos casos ya gozan de autonomía plena. Los procedimientos de acreditación son comunes para las universidades públicas y privadas. 
Cada país organiza su sistema de aseguramiento de la calidad de manera diferente. Argentina concentra todos los procesos en una sola agencia: Comisión Nacional de Investigación de Acreditación y Evaluación Universitaria, CONEAU. Chile comenzó por la autorización de instituciones y programas del sector privado en 1990 y, más adelante, introdujo la acreditación, usando diversas agencias del Estado. Recientemente, ha aprobado una ley que unifica los procesos en un sistema de aseguramiento de la calidad. La regulación uruguaya estableció procedimientos de autorización institucional y de programas para las instituciones privadas y ha comenzado a implementar procesos de acreditación en el marco del proceso experimental del MERCOSUR, que permite la participación de la universidad pública.

El argumento principal del artículo se concentra en la interacción entre las tendencias reseñadas, y sostiene que los mecanismos de aseguramiento de la calidad juegan dos roles contradictorios respecto del desarrollo de la educación superior privada. Por un lado, el establecimiento de mecanismos de autorización institucional y de programas limita la proliferación privada. Los escenarios observados antes y después de la creación de las agencias responsables del otorgamiento de las autorizaciones son completamente diferentes. Por otra parte, una vez que las instituciones han sido autorizadas, se produce una segunda onda del impulso privado, especialmente en los países que conceden una autonomía completa a las universidades privadas, permitiendo que abran nuevos programas y sedes en diversas localidades, como en el caso de Argentina y Chile. En algunas oportunidades, la acreditación actúa incluso como impulso dinámico en términos de la legitimidad de las instituciones privadas. Los casos argentino, chileno y uruguayo proporcionan evidencias relevantes sobre el impacto de los mecanismos de aseguramiento de la calidad en el desarrollo de la educación superior privada. 


\section{Evolución de la educación superior privada en América Latina}

\section{El desarrollo del sector privado}

Los tres países objeto de este estudio comparten los mismos orígenes en términos del desarrollo de la educación superior. Como nuevas repúblicas independientes, en la primera mitad del siglo XIX sintieron la necesidad de crear universidades públicas nacionales como parte del esfuerzo de construcción nacional y con el objeto de preparar a las elites profesionales que gobernarían los nuevos países. La Universidad de Buenos Aires (1821), la Universidad de Chile (1842) y la Universidad de la República (1836) fueron parte de la misma tendencia de universidades nacionales fundadas, controladas y financiadas por el Estado. El creciente secularismo distinguió a las universidades nacionales de ejemplos coloniales anteriores. La función principal de las nacionales era -y probablemente sigue siendo- la preparación profesional. Estas universidades gozaron del monopolio de conceder grados académicos, instrumento importante de avance social.

El desarrollo privado de la educación superior siguió diversas trayectorias en los tres países. Chile fue el primer país en América Latina que permitió instituciones privadas en 1888, con la creación de la Pontificia Universidad Católica; en Argentina, la primera universidad privada fue autorizada en 1959 y Uruguay las permitió recién en 1985.

Levy (1986) proporciona un modelo analítico para entender el desarrollo de la educación superior privada en América Latina, describiendo tres olas de la privatización en los sistemas de la región

La primera ola de reacción a la dominación pública en la educación superior fue encabezada por la Iglesia Católica. En la mayoría de los países de la región, las primeras universidades privadas fueron católicas, incluyendo los casos de Argentina, Chile y Uruguay. 
Sin embargo, casi hay un siglo entre la creación de la Pontificia Universidad Católica en Chile (1888) y de la Universidad Católica del Uruguay (1985). La razón principal que movilizó a la Iglesia Católica para crear universidades fue la secularización del Estado y de la sociedad que se reflejaba (o era el fruto de) las universidades estatales. Las universidades católicas se propusieron intervenir en el debate público y en la formación de las clases dirigentes. Sus esquemas de organización y los campos del estudio en que se desarrollaron no rompieron con los modelos de las universidades públicas.

El concepto de fracaso del sector público es utilizado por Levy (1986) para explicar el cambio empírico de preferencias entre los sectores y que justifica las dos siguientes olas. La expansión de la matrícula es un rasgo típico de los sistemas de educación superior de América Latina. El aumento del número de estudiantes provocó gran tensión en las universidades públicas para absorber la nueva demanda. Las instituciones recibieron fuerte presión para democratizar la educación superior, forzándolas a abrirse a una clase media cada vez mayor y perdiendo su naturaleza de elite. La tabla 1 muestra el crecimiento de la matrícula y de la tasa de matriculación en los países estudiados entre los años 1950 y 2000.

Tabla 1: Crecimiento de la matrícula en Argentina, Chile y Uruguay.

\begin{tabular}{l|r|c|c|c|c}
\hline & \multicolumn{2}{|c|}{1950} & \multicolumn{2}{c|}{2000} & Crecimiento \\
\hline País & Matrícula & Tasa & Matrícula & Tasa & \\
\hline Argentina & 82.531 & $5,2 \%$ & 1.269 .239 & $36,0 \%$ & $1,438 \%$ \\
\hline Chile & 9.528 & $1,7 \%$ & $452.347(*)$ & $26,7 \%$ & $4,648 \%$ \\
\hline Uruguay & 11.722 & $6,0 \%$ & 79.678 & $32,5 \%$ & $580 \%$ \\
\hline
\end{tabular}

Fuentes: Para 1950, Schwartzman (1994); para 2000, Argentina: Fernández Lamarra (2003); Chile: Bernasconi (2003), y Uruguay: AEE (2000).

${ }^{*}$ Incluye estudiantes de institutos universitarios y profesionales.

El aumento de la matrícula y la politización de universidades públicas producen la segunda ola de crecimiento privado, con la creación de universidades de elite como reacción. En los años 60 y 
comienzo de los 70, las públicas sufrieron la inquietud estudiantil con interrupciones frecuentes de sus servicios. Durante esos años se crearon universidades de elite que permitieron a quienes podían pagar por sus estudios la realización de carreras universitarias sin las complicaciones de las universidades públicas. Algunas de estas instituciones eran afiliadas a entidades religiosas, pero la mayoría no. Las universidades de elite se caracterizaron por brindar condiciones adecuadas para el aprendizaje. Los factores económicos y políticos confluyeron para impulsar el crecimiento privado basado en las carencias percibidas en el sector público.

Los problemas en las universidades públicas tradicionales no fueron un fenómeno exclusivo de América Latina, dado que en Europa y Estados Unidos se produjeron también deterioros importantes de sus condiciones académicas. Sin embargo, la mayoría de las naciones desarrolladas adoptaron reformas estructurales democratizando los procesos de toma de decisiones académicas y abriendo las instituciones a la influencia externa, tanto por parte de los gobiernos como de los sectores productivos y de la sociedad civil (Altbach, 2005). Las universidades públicas latinoamericanas fueron lentas en cambiar.

Una tercera ola de instituciones privadas refiere a las universidades que fueron creadas como resultado del fracaso del sector público en absorber la creciente demanda de educación superior. Levy llama a estas instituciones de "absorción de demanda" porque proporcionan una alternativa privada a instituciones públicas inaccesibles o poco atractivas. En los países que decidieron proteger sus universidades públicas con difíciles exámenes de ingreso, como Brasil o Costa Rica, las instituciones de absorción de demanda han florecido.

La proliferación privada ha sido una característica común de los países en estudio en las décadas de 1980 y comienzo de los 90, tomando por sorpresa a los gobiernos. El inesperado crecimiento privado produjo la acción reactiva del Estado dando por resultado nuevos sistemas de regulación. 


\section{Peculiaridades de cada uno de los países}

Las tres olas del desarrollo privado son observables en Argentina, Chile y Uruguay, aunque con características diferentes.

El monopolio del sector público duró en Argentina hasta 1959, cuando una ley autorizó la creación de universidades privadas. Las primeras cuatro fueron entidades católicas; en la siguiente década se establecieron 24 universidades privadas, de las cuales 10 eran católicas (Cosentino de Cohen, 2003). Durante los 90 se produjo un proceso de proliferación institucional, como respuesta a la creciente demanda, fomentándose la diversificación del sistema. El sector privado experimentó un importante crecimiento, autorizándose 23 universidades entre 1990 y 1995. La proliferación institucional también se dio en el sector público, donde nueve fueron creadas en el mismo periodo (Fernández Lamarra, 2003). La tabla 2 sintetiza los cambios en el número de instituciones universitarias por sector.

Tabla 2: Proliferación institucional en Argentina.

\begin{tabular}{l|c|c}
\hline & 1970 & 2003 \\
\hline Público & 15 & 45 \\
Universidades & & 39 \\
Institutos universitarios & & 6 \\
\hline Privado & 24 & 54 \\
Universidades & 42 \\
Institutos universitarios & 39 & 12 \\
\hline Total & & 99 \\
\hline
\end{tabular}

Fuentes: Para 1970: Cosentino (2003 p.10); para 2003: Caillón (2005 p. 6).

En Argentina, la regulación y el financiamiento de la educación superior son diferentes para el sector público y el privado. Las instituciones privadas no reciben financiamiento público y para funcionar precisan de una autorización estatal; las públicas son subvencionadas totalmente por el Estado y los estudiantes no pagan aranceles, a excepción de algunos estudios de posgrado. La matrícula de la educación superior en Argentina ha crecido un 89\% entre 1993 y 2003 (Caillón, 2005). El sector privado representó el 14,4\% de los matriculados en 2003. 
Las olas II e III se dieron de manera simultánea en Argentina en la década del 90, debido a que se produjeron procesos de masificación y proliferación en el sector, pero, de todos modos, algunas de estas universidades mantuvieron su prestigio. En consecuencia, el sector privado creció pero de manera limitada, distinguiéndose emprendimientos que se pueden calificar como "de elite" de otros que configuran más bien el tipo de instituciones de "absorción de demanda". Existe una creciente influencia del sector privado como consecuencia de un incremento en las inscripciones de posgrado y en el porcentaje de graduados. Las universidades privadas proveyeron el 25\% de los graduados universitarios totales del año 2003 (Caillón, 2005). Sin embargo, la ausencia de fondos públicos de financiamiento para estos programas produjo que las instituciones del sector privado se concentraran en las áreas urbanas del país y ofrecieran programas en áreas de amplia demanda y que requieren de una inversión de capitales más baja.

Hasta el año 80, la educación superior en Chile era básicamente un esfuerzo público, dado que las dos universidades estatales y seis universidades privadas (tres de ellas católicas) eran financiadas por el Estado. La temprana autorización de la Pontificia Universidad Católica de Chile (1888) es un ejemplo de la primera ola de reacción a la dominación pública. El regionalismo fue un factor clave en Chile para el desarrollo privado, dado que se autorizaron universidades privadas en Concepción, Valparaíso, Valdivia y Antofagasta, entre 1919 y 1956.

La reforma de 1981 cambió dramáticamente la educación superior chilena, al introducir el pago de aranceles para todos los estudios universitarios y otros mecanismos de competencia, así como la fragmentación de las universidades públicas. El resultado de la nueva regulación fue la proliferación institucional (Bernasconi, 2003). En 1990 fue aprobada una ley que creó mecanismos de regulación para la autorización institucional y de programas, limitando el crecimiento del número de instituciones: desde esa fecha solamente 10 universidades privadas fueron autorizadas y, por otra parte, 13 
fueron cerradas (Lemaitre, 2005). La tabla 3 muestra los cambios que la educación superior chilena experimentó en términos del número de instituciones.

Tabla 3: Proliferación institucional en Chile

\begin{tabular}{l|c|c}
\hline Universidades & 1980 & 2003 \\
\hline Públicas & 2 & 16 \\
Privadas tradicionales (financiadas públicamente) & 6 & 9 \\
Nuevas privadas & & 38 \\
Total & 8 & 63 \\
\hline
\end{tabular}

Fuente: Bernasconi (2003).

Las universidades privadas tradicionales creadas antes de la reforma se regulan de la misma manera que las instituciones públicas, porque reciben un subsidio directo y tienen estatuto jurídico autónomo. Las nuevas universidades privadas tienen que presentarse a un proceso de evaluación por parte del Consejo Superior de Educación (CSE) para obtener su autorización. Por un periodo de entre seis y once años, sus actividades están bajo supervisión; por lo tanto las universidades privadas no gozan de la autonomía académica completa. De las 38 universidades privadas existentes, 24 han alcanzado su autonomía (Lemaitre, 2005). En términos del financiamiento, estas instituciones reciben solamente un subsidio indirecto para el porcentaje de estudiantes que ingresan con un alto rendimiento en la Prueba de Selección Universitaria, PSU.

El proceso de reforma tuvo profundo impacto en el sistema de educación superior chilena. Las instituciones se diversificaron y las inscripciones crecieron hasta 142\% en una década. El patrón de financiamiento del sistema fue cambiado de la oferta a la demanda incluyendo becas y préstamos. Todas las universidades chilenas (públicas y privadas) se han comprometido en comportamientos competitivos tanto por estudiantes como por profesores (Bernasconi, 2005), guiados por las fuerzas del mercado (Brunner, 2005). El sector privado ha contribuido decisivamente en este proceso, 
272 ASEGURAMIENTO DE LA CALIDAD Y DESARROLLO DE LA EDUCACIÓN SUPERIOR PRIVADA - Pablo Landoni, Carlos Romero

correspondiéndole un 59\% de la matrícula en el año 2000 (Bernasconi, 2003).

Uruguay fue el último país latinoamericano (con excepción de (uba) en permitir el establecimiento de instituciones privadas en educación superior. Por un tiempo, el país decidió absorber el aumento de la demanda en su única universidad pública. A principios de los 80 el gobierno militar adoptó exámenes de ingreso y políticas de cuotas para limitar la demanda. Cuando la democracia volvió al país (1985), las políticas de acceso abierto fueron restauradas y la matrícula se expandió de 27.000 en 1983 a 61.000 en 1988. La explosión de la demanda fue difícil de manejar por la Universidad de la República.

Por 150 años, la universidad nacional gozó del monopolio en la educación superior (Romero y Landoni, 2001). En 1985 la Universidad Católica de Uruguay fue autorizada a funcionar y, diez años más tarde, una nueva regulación fue aprobada para el reconocimiento de instituciones y programas privados. Desde 1995 se han establecido cuatro universidades y 11 institutos universitarios en el sector privado.

Las tres olas discutidas por Levy se iniciaron casi simultáneamente en Uruguay. Sin embargo, hubo intentos de creación de universidades católicas a fines del siglo XIX (contemporáneos a la creación de la de Chile) y a principios de los 60 (simultáneos a la creación de la Católica de Argentina) (Monreal, 2005).

El sistema universitario uruguayo es dual. Por una parte, la Universidad de la República es la única universidad pública con un régimen de autonomía completa: es subsidiada por fondos del presupuesto público, enfrenta importantes problemas de masificación porque tiene un sistema de ingreso libre y no carga aranceles salvo en programas de posgrado de corte profesional. Por otra, las instituciones privadas de educación superior son supervisadas por el Ministerio de Educación y no reciben financiamiento del Estado, ni como subsidio 
ni por becas o préstamos a estudiantes. El sector privado contribuye con un $12,3 \%$ de la matrícula universitaria.

En síntesis, los casos de Argentina, Chile y Uruguay confirman las tendencias globales de crecimiento del sector privado. En los tres países se ha dado un aumento continuo de inscripciones en instituciones universitarias privadas, en un contexto de incremento del total de estudiantes matriculados en la educación superior, como lo refleja la tabla 4.

Tabla 4: Porcentajes de crecimiento de la matrícula en los países objeto de estudio.

\begin{tabular}{lccc}
\hline & $\begin{array}{c}\text { Crecimiento de la } \\
\text { matrícula privada en la } \\
\text { última década }\end{array}$ & $\begin{array}{c}\text { Crecimiento de la } \\
\text { matrícula total en la } \\
\text { última década }\end{array}$ & $\begin{array}{c}\text { Porcentaje actual de la } \\
\text { matrícula privada }\end{array}$ \\
\hline Argentina & $91 \%$ & $89 \%$ & $14,4 \%$ \\
Chile & $182 \%$ & $142 \%$ & $59,0 \%$ \\
Uruguay & $356 \%$ & $24 \%$ & $12,3 \%$ \\
\hline
\end{tabular}

Fuente: Elaboración propia sobre la base de Caillón 2005 para Argentina, Bernasconi 2003 para Chile y AEE para Uruguay.

\section{El desarrollo de mecanismos de aseguramiento de la calidad en el Cono Sur}

Los fenómenos de la masificación y sus consecuencias sobre la proliferación y diversificación de la educación superior, incluyendo el desarrollo del sector privado, han obligado al Estado a introducir nuevos instrumentos de regulación, entre los que se encuentran los de aseguramiento de la calidad (Levy, 2006). Las sociedades han perdido confianza en estas instituciones y demandan una rendición de cuentas (accountability) por sus acciones.

En el caso de Argentina, en 1995 se creó la Agencia Nacional de Evaluación y Acreditación (CONEAU), que tiene como misión la evaluación institucional de universidades con estatuto autónomo, la acreditación institucional de universidades e institutos universitarios privados creados después de 1990 y la acreditación de programas, tanto en el nivel de grado como de posgrado, para los sectores público 
274 ASEGURAMIENTO DE LA CALIDAD Y DESARROLLO DE LA EDUCACIÓN SUPERIOR PRIVADA - Pablo Landoni, Carlos Romero

y privado (Fernández Lamarra, 2004). Argentina optó por un modelo de la concentración de funciones en CONEAU, lo que, en la práctica, ha llevado a que se dilaten algunas de sus actividades, especialmente en la acreditación de programas (Fernández Lamarra 2003).

Chile ha aprobado, en julio de 2006, una ley que establece un Sistema Nacional de Aseguramiento de la Calidad de la Educación Superior. A diferencia del caso argentino, la regulación chilena ha diversificado estas tareas en diversas agencias, aunque el nuevo marco legal le da una articulación sistémica mayor. El licenciamiento de nuevas instituciones sigue a cargo del CSE, regulado por la Ley Orgánica Constitucional de Enseñanza, LOCE, de 1990. La acreditación institucional, como proceso de análisis de los mecanismos existentes al interior de las instituciones autónomas de educación superior para asegurar su calidad, será responsabilidad de la Comisión Nacional de Acreditación (CNA). La de carreras o programas, tanto de grado como de posgrado, será realizada por agencias acreditadoras, autorizadas por la CNA, sustituyendo las funciones asignadas en 1998 a la Comisión Nacional de Acreditación de Pregrado (CNAP) y a la Comisión Nacional de Acreditación de Posgrado (CONAP). Las instituciones reconocen que este proceso ha mejorado la calidad del sistema de educación superior chileno, aunque coinciden en que todavía hay que cambiar la cultura académica e introducir el aseguramiento de la calidad como instrumento relevante de la gestión universitaria (Lemaitre, 2005).

La regulación uruguaya distingue claramente entre el sector público y el privado. La Universidad de la República es completamente autónoma, tanto para el otorgamiento de grados académicos como para la creación de programas de estudios. Por el contrario, las instituciones privadas y sus programas requieren de la autorización del Ministerio de Educación, asesorado por el Consejo Consultivo de la Enseñanza Terciaria Privada (CCETP). En materia de acreditación, solamente se ha realizado un ejercicio experimental en el marco del Mecanismo de Acreditación definido por el Mercado Común del Sur (MERCOSUR) (Landoni y Martínez, 2006). 
La tabla 5 resume los sistemas de aseguramiento de la calidad disponibles en cada uno de los países y sus características específicas.

Tabla 5: Sistemas de aseguramiento de la calidad.

\begin{tabular}{|c|c|c|c|c|c|c|}
\hline \multirow[b]{2}{*}{ Nivel } & \multicolumn{2}{|c|}{ Argentina } & \multicolumn{2}{|c|}{ Chile } & \multicolumn{2}{|c|}{ Uruguay } \\
\hline & Agencia & Aplicación & $\begin{array}{c}\text { Agencias / } \\
\text { Estado }\end{array}$ & Aplicación & $\begin{array}{c}\text { Agencias / } \\
\text { Estado }\end{array}$ & Aplicación \\
\hline $\begin{array}{l}\text { Autorización, } \\
\text { licenciamiento o } \\
\text { reconocimiento } \\
\text { instituciones } \\
\text { privadas }\end{array}$ & $\begin{array}{l}\text { CONEAU } \\
\text { (obligatorio) }\end{array}$ & $\begin{array}{l}\text { Instituciones } \\
\text { privadas }\end{array}$ & $\begin{array}{l}\text { CSE } \\
\text { (obligatorio) }\end{array}$ & $\begin{array}{l}\text { Instituciones } \\
\text { privadas }\end{array}$ & $\begin{array}{l}\text { MEC con } \\
\text { asesoramiento } \\
\text { CCETP } \\
\text { (obligatorio) }\end{array}$ & $\begin{array}{l}\text { Instituciones } \\
\text { privadas }\end{array}$ \\
\hline $\begin{array}{l}\text { Acreditación } \\
\text { (evaluación) } \\
\text { institucional }\end{array}$ & $\begin{array}{l}\text { CONEAU } \\
\text { (voluntario) }\end{array}$ & $\begin{array}{l}\text { Público y } \\
\text { privado } \\
\text { (autonomía) }\end{array}$ & $\begin{array}{l}\text { CNA } \\
\text { (voluntario) }\end{array}$ & $\begin{array}{l}\text { Público y } \\
\text { privado } \\
\text { (autonomía) }\end{array}$ & & $\begin{array}{l}\text { No } \\
\text { disponible }\end{array}$ \\
\hline $\begin{array}{l}\text { Acreditación } \\
\text { de programas } \\
\text { académicos de } \\
\text { grado }\end{array}$ & $\begin{array}{l}\text { CONEAU } \\
\text { (obligatorio) }\end{array}$ & $\begin{array}{l}\text { Público y } \\
\text { privado } \\
\text { (sólo } \\
\text { profesionales } \\
\text { regulados por } \\
\text { ley) }\end{array}$ & $\begin{array}{l}\text { Agencias } \\
\text { acreditadoras. } \\
\text { Antes CNAP } \\
\text { (voluntario, } \\
\text { salvo carreras } \\
\text { definidas por } \\
\text { ley) }\end{array}$ & $\begin{array}{l}\text { Público y } \\
\text { privado }\end{array}$ & $\begin{array}{l}\text { Comisión } \\
\text { ad hoc } \\
\text { (voluntario) }\end{array}$ & $\begin{array}{l}\text { MERCOSUR } \\
\text { (3 programas) }\end{array}$ \\
\hline $\begin{array}{l}\text { Acreditación } \\
\text { de programas } \\
\text { académicos de } \\
\text { posgrado }\end{array}$ & $\begin{array}{l}\text { CONEAU } \\
\text { (obligatorio) }\end{array}$ & $\begin{array}{l}\text { Público y } \\
\text { privado }\end{array}$ & $\begin{array}{l}\text { Agencias } \\
\text { acreditadoras } \\
\text { Antes CONAP } \\
\text { (voluntario) }\end{array}$ & $\begin{array}{l}\text { Público y } \\
\text { privado }\end{array}$ & & No disponible \\
\hline
\end{tabular}

Una característica importante de la acreditación en todos los países analizados es que ha sido diseñada como un mecanismo para el conjunto del sistema, incluyendo instituciones y programas, tanto públicos como privados. Está disponible en Argentina y Chile, pero no en Uruguay. Se ha producido en los países un debate acerca del impacto de la evaluación institucional sobre la autonomía de las universidades. Las universidades son celosas de su autonomía y no estarían inclinadas a participar en los procesos de acreditación si ésta se viera comprometida. Este hecho es evidente en el caso argentino donde se ha usado la denominación "evaluación institucional" y no "acreditación" por respeto a la autonomía, en particular de las instituciones públicas. 


\section{Impactos de los mecanismos de aseguramiento de la calidad en el desarrollo de la educación superior privada}

La implementación de estos mecanismos está produciendo impactos importantes en los sistemas de educación superior de Argentina, Chile y Uruguay. La preguntas clave de este estudio son cómo las instituciones privadas reaccionan a estos cambios y cuáles son los comportamientos esperados por parte de los diversos actores del sistema. Para ello se consideran dos fuerzas que influyen sobre los sistemas de educación superior: el isomorfismo y la diversificación. Se observan tres tipos de isomorfismo en los sistemas universitarios: el coercitivo, que viene generalmente del Estado, el normativo, provocado por la cultura profesional dominante, y el imitativo, por el cual las instituciones copian voluntariamente a las organizaciones dominantes (Levy, 1999). El estudio del impacto de estas fuerzas sobre los sistemas universitarios contribuye a entender las acciones de las instituciones privadas y las reacciones por parte del Estado.

Tres impactos principales se han identificado en los estudios de caso, como consecuencia de la introducción de mecanismos de aseguramiento de la calidad en el desarrollo privado:

El primero fue que los procesos de autorización (licenciamiento o reconocimiento según los países) restringieron la proliferación de las instituciones privadas. Las agencias responsables de evaluar nuevas ofertas fueron rigurosas en los procesos de aprobación; por ejemplo, CONEAU aprobó solamente nueve de las 83 ofertas presentadas en la década pasada (Fernández Lamarra, 2003). La tabla 6 corrobora con cifras el impacto de la acción estatal en los procesos de autorización de las instituciones privadas luego de creadas las agencias especializadas. 
Tabla 6: Impactos de los mecanismos de aseguramiento privado en la proliferación institucional privada.

\begin{tabular}{l|c|c}
\hline & $\begin{array}{c}\text { Instituciones creadas antes de } \\
\text { la agencia }\end{array}$ & $\begin{array}{c}\text { Instituciones autorizadas por } \\
\text { la agencia }\end{array}$ \\
\hline $\begin{array}{l}\text { Argentina } \\
\text { CONEAU (1995) }\end{array}$ & 22 & 9 \\
\hline Chile & $(1990-1995)$ & 10 \\
CSE (1990) & 40 & 14 \\
\hline Uruguay & $(1985-1995)$ & 14 \\
CCETP (1995) & 1 & \\
\hline
\end{tabular}

Fuente: Elaboración propia sobre la base de Fernández Lamarra 2004, Lemaitre 2005 y Landoni y Martínez 2006.

En los procesos de autorización institucional se observan casos de isomorfismo coercitivo por parte del Estado o de las organizaciones dominantes. En los casos de Argentina y Uruguay, un estándar clave para reconocer a una entidad privada es que tenga las características propias de una organización universitaria. La pregunta es si existe una noción fija de "universidad" y cuál es el modelo para definirla. La literatura en esta materia demuestra que las universidades privadas traen diversidad a los sistemas universitarios en términos de misiones, objetivos y organización (Levy, 2004). Por lo tanto, las agencias utilizan a veces isomorfismo coactivo para limitar la capacidad de las instituciones de ser distintas en maneras que no están listas para aprobar.

Un segundo hallazgo es que la acreditación ha tenido un limitado impacto en el crecimiento y en producir homogeneidad en las instituciones privadas. Las inscripciones en este sector continuaron creciendo a pesar del establecimiento de mecanismos de acreditación, beneficiándose todavía de los fracasos en las instituciones públicas. La acreditación está teniendo efectos positivos al introducir la cultura de la autoevaluación en las instituciones y podría ayudar a restaurar la confianza dentro de las universidades públicas masificadas. Sin embargo, no se observan consecuencias significativas de la acreditación en las tendencias generales de matriculación. No está claro si está produciendo efectos en materia de homogeneizar la oferta privada 
debido a un uso rígido de estándares. Las agencias de acreditación han sido cuidadosas en la definición de éstos, respetando la diversidad de misiones y objetivos de las universidades; sin embargo, existen evidencias de isomorfismo normativo en el comportamiento de los pares evaluadores, en la medida en que utilizan valores tradicionales de la profesión y de la cultura académica dominantes, que en la región todavía son los provenientes de las universidades públicas (Fernández Lamarra, 2004; Landoni y Martínez, 2006).

A veces la homogeneización es resultado más de estrategias institucionales que de consecuencias directas de la regulación (Van Vught, 1996). Las instituciones menos prestigiosas intentan alcanzar mayor reputación mediante la obtención de la acreditación. Un caso común de isomorfismo imitativo proviene de la contratación, por parte de las instituciones privadas, de académicos formados en las tradicionales universidades públicas de los países (Levy, 1999). En Argentina y Uruguay, debido a las escasas contrataciones por tiempo completo, los cuerpos docentes suelen ser mano de obra compartida. Para acceder a mejores remuneraciones (y generalmente para evitar el trabajo profesional), es un comportamiento racional por parte de los académicos enseñar tanto en universidades públicas como en privadas simultáneamente.

Un tercer impacto es que las instituciones privadas incluyeron la acreditación en su estrategia para obtener legitimidad. Estas universidades han sido activas en solicitar ser acreditadas. En Uruguay, en el caso de acreditación de programas de Ingeniería usando el mecanismo del MERCOSUR, la mitad de los programas evaluados provienen del sector privado. En Argentina, en el caso de Medicina, 16 de los 28 programas que solicitaron la evaluación pertenecen a instituciones privadas (Caillón, 2005).

La acreditación funciona como factor externo que afecta el ambiente de las instituciones de educación superior (Meek, 1996). Las universidades privadas en Argentina, Chile y Uruguay han sido rápidas en ajustarse a las políticas estatales y la participación en 
procedimientos de acreditación ha sido parte de su estrategia de legitimación (Lemaitre, 2005). Como excepciones se encuentran los casos de algunas universidades privadas tradicionales en Chile, ya prestigiosas, que no tienen ninguna necesidad de la acreditación como ventaja para atraer a estudiantes. La legitimidad es muy importante para las universidades privadas pues estimula su competitividad en el mercado de la educación superior.

Los estudios de casos demuestran que la acreditación está atenuando la división entre los diferentes sectores en sistemas fuertemente diferenciados en términos de regulación y financiamiento, como Argentina y Uruguay. La proliferación institucional en Argentina requirió la introducción de mecanismos para asegurar la calidad en el conjunto del sistema. El caso uruguayo mantiene su característica de predominio del sector público sobre el privado, a veces innovando hacia la diversificación, en otras optando por crecimiento no diferenciado imitando a las instituciones más prestigiosas. El proceso de reforma que comenzó en 1980 cambió significativamente el sistema superior chileno. Sin embargo, mantuvo sus rasgos de homogeneidad entre los sectores público y privado, aunque ahora mucho más orientado hacia el mercado (Brunner, 2005).

\section{Reconocimientos}

A Andrés Bernasconi y Cynthia Jeppesen por compartir sus conocimientos sobre los sistemas de educación superior de Argentina y de Chile.

\section{Referencias bibliográficas}

AEE Anuario Estadístico de Educación. Ministerio de Educación y Cultura. Años 1995 al 2004. Montevideo: MEC.

Altbach, Philip (1999) Perspectives on Private Higher Education. In: Altbach, P. (ed.) Private Prometheus: Private Higher Education and Development in the $21^{\text {st }}$ Century. Westport: Greenwood.

Altbach, Philip (2005) Patterns in Higher Education Development. In: Altbach, 
280 ASEGURAMIENTO DE LA CALIDAD Y DESARROLLO DE LA EDUCACIÓN SUPERIOR PRIVADA - Pablo Landoni, Carlos Romero

P.; Berdahl, R. y Gumport, P. (eds.) American Higher Education in the Twenty-First Century: Social, Political and Economic Challenges. Baltimore: The Johns Hopkins University Press.

Bernasconi, Andrés (2003) Informe sobre la Educación Superior en Chile: 19802003. UNESCO-IESALC. Disponible en: http://www.iesalc.unesco. org.ve

Bernasconi, Andrés (2005) Incentives for Faculty Performance and the Consolidation of the Academic Profession: Chile 1960-2000. Presentado en el seminario de Fulbright "Quality and Equity in Education Reform", marzo 2005, Boston: LASPAU y Harvard Graduate School of Education.

Brunner, José J. (2005) Informe sobre la Educación Superior en Chile: Guiar el Mercado. Santiago de Chile: Universidad Adolfo Ibáñez, Escuela de Gobierno.

Caillón, Adriana (2005) La Educación Superior Universitaria Privada en Argentina. UNESCO-IESALC, pp. 61-63. Disponible en: http://www. iesalc.unesco.org.ved

Cosentino de Cohen, Clemencia (2003) Diversification in Argentine Higher Education: Dimensions and Impact of Private Sector Growth Higher Education, 46, pp. 1-35.

Fernández Lamarra, Norberto (2003) La Educación Superior Argentina en Debate: Situación, problemas y perspectivas. Buenos Aires: IESALCUNESCO/EUDEBA, p. 39.

Fernández Lamarra, Norberto (2004) Evaluación y Acreditación de la Educación Superior en la Educación Superior Argentina. En: La Evaluación y la Acreditación en la Educación Superior en América Latina y el Caribe. UNESCO-IESALC, pp. 15-30. Disponible en: http://www. iesalc.unesco.org.ve/estudios/regionales_lat/evalyacredalc.pdt

Landoni, Pablo y Martínez, Enrique (2006) La Institucionalidad de la Acreditación: ¿Hacia dónde vamos? La Autonomía Universitaria y el rol del Ministerio de Educación. Disponible en: http://www.iesalc.unesco. org.ve/pruebaobservatorio/documentos.pdf/seminarid

Lemaitre, María José (2005) Aseguramiento de la Calidad en Chile: Impacto y Proyecciones. Presentado para el X Seminario Internacional "Aseguramiento de la Calidad: Impacto y Proyecciones", septiembre 2005, Santiago de Chile: CSE y CNAP, pp. 11-14. 
Levy, Daniel (1986) Higher Education and the State in Latin America: Private Challenges to Public Dominance. Chicago: University of Chicago Press, p. 37.

Levy, Daniel (1999) When Private Higher Education Does Not Bring Organizational Diversity: Argentina, China, Hungary. In: Altbach, P. (ed.) Private Prometheus: Private Higher Education and Development in the $21^{\text {st }}$ Century. Westport: Greenwood.

Levy, Daniel (2002) Unanticipated Development: Perspectives on Private Higher Education's Emerging Roles. PROPHE (Program for Research on Private Higher Education) Trabajo \# 1. Disponible en: http://www.albany. edu/ prophe/publication.htm

Levy, Daniel (2004) The New Institutionalism: Mismatches with Private Higher Education's Global Growth. PROPHE (Program for Research on Private Higher Education) Trabajo \# 3. Disponible en: http://www.albany. edu/ prophe/publication.htm

Levy, Daniel (2006) The Private Fit in the Higher Education Landscape. In Forest, J. and Altbach, P. (eds.) International Handbook of Higher Education Dordrecht: Springer.

Meek, L.; Goedegebuure, L. and Kivinen O. (eds.) 1996. The Mockers and the Mocked: Comparative Perspectives on Differentiation, Convergence and Diversity in Higher Education. Surrey: Pergamon.

Monreal, Susana (2005) Universidad Católica del Uruguay: El largo Camino hacia la Diversidad. Montevideo: UCU.

Romero, Carlos y Landoni, Pablo (2001) Educación Superior Uruguaya: del Monopolio a la Consolidación del Sistema. En: Martinic, Sergio y Pardo, Marcela. Economía Política de las Reformas Educativas en América Latina. Santiago de Chile: CIDE/PREAL.

Schwarz, Stefanie and Westerheijden, Don (2004) Accreditation in the Framework of Evaluation Activities: A comparative study in the European Higher Education Area. In: Schwarz, Stefanie and Westerheijden Don (eds.) Accreditation and Evaluation in the European Higher Education Area. Kluwer: Dordrecht.

Schwartzman, Simón (1994) Las Universidades Latinoamericanas en Contexto. En Malo, Salvador y Morley, Samuel (eds.) La Educación Superior en América Latina: Testimonios de un Seminario de Rectores. Washington DC: BID-UDUAL. 
282 ASEGURAMIENTO DE LA CALIDAD Y DESARROLLO DE LA EDUCACIÓN SUPERIOR PRIVADA - Pablo Landoni, Carlos Romero

Van Vught, Frans (1996) Isomorphism in Higher Education? Towards a Theory of Differentiation on Diversity in Higher Education Systems. Meek, L.; Goedegebuure, L. and Kivinen O. (eds.) 1996. The Mockers and the Mocked: Comparative Perspectives on Differentiation, Convergence and Diversity in Higher Education. Surrey: Pergamon.

Recibido: 31 de octubre de 2006

Aceptado: 14 de noviembre de 2006 